\title{
Editorial to the topical issue "Advanced Metamaterials in Microwaves, Optics and Mechanics"
}

\author{
Christos Argyropoulos ${ }^{1, *}$ and Alessio Monti ${ }^{2, *}$ \\ 1 University of Nebraska-Lincoln, Department of Electrical and Computer Engineering, 16th St., Lincoln, NE 68588-0511, USA \\ 2 Niccolò Cusano University, Via don Carlo Gnocchi 3, 00166 Rome, Italy
}

\begin{abstract}
This editorial inaugurates the first edition of the Special Issue of the Metamaterials' Congress published on EPJ Applied Metamaterials. The special issue was born by a partnership between the Editor in Chief of EPJApplied Metamaterials - Prof. Yang Hao - and the Congress General Chairs - Profs. Filiberto Bilotti and Andrea Alù. The 16 extended papers available in this first edition have been selected among over 420 contributions accepted at the Metamaterials'2015 Congress with the aim to provide a representative sample of the scientific topics dealt during the days of the Congress in the city of Oxford at United Kingdom.
\end{abstract}

The introduction of artificial materials and metamaterials in the last 15 years represented an important breakthrough in the research fields of applied and theoretical electromagnetics. The possibility to control at will the response of a bulk macroscopic material by acting on its microscopic constituents allowed the birth of an extremely dynamic and interdisciplinary research field whose effective extent is not yet evident. In fact, even though the systematic research about these topics can be dated back to 2000, when the first paper on double-negative metamaterials has been published, the research trends on metamaterials are currently experiencing an ever-growing development both in their original discipline, i.e. electromagnetics and optics, and in other scientific and technological fields, such as mechanics, acoustics, thermodynamics, quantum theory, etc. This is confirmed by the number of publications relevant to metamaterials indexed in the international databases. Scopus, for example, counts about 11000 publications on metamaterials in the last 5 years of which about 2000 are mechanic or acoustic metamaterial papers.

In this editorial, we introduce the inaugural Special Issue of the Metamaterials Congress, which is planned to be published annually and it is born from a partnership between the EPJ Applied Metamaterials (EPJ-AM) journal and the International Congress on Advanced Electromagnetic Materials in Microwaves and Optics. The last edition of the conference, organized by the Virtual Institute for Artificial Electromagnetic Materials and Metamaterials (Metamorphose VI AISBL), comprised a 4-day Conference (7-10 September 2015) and a 2-day Doctoral School (11-12 September 2015). The congress took place in the wonderful city of Oxford in United Kingdom and saw the participation of about 430 delegates from 40 different countries from all over the world. Due to the extraordinary number of high-quality submissions, this edition required four parallel sessions rather than the usual three, confirming the continual growth of the international metamaterials community.

We are very glad to serve as Guest-Editors in the first edition of this Special Issue that contains a variety of regular and review invited articles selected among the papers submitted to Metamaterials'2015 congress. The aim of the Special Issue was to provide the possibility to extend in a journal paper a representative collection of the best contributions submitted and presented during the Congress. In the selection process, we have taken into account the new fascinating trends in the metamaterial research without neglecting the more mature topics some of which are currently evolving towards new technological achievements. After the selection process, we succeeded to select 16 papers (13 regular papers and 3 reviews) that, in their heterogeneity, are able to provide a fresh overview of some of the most important topics and scientific discussions occurred during the last edition of the conference.

In more details, the paper by $\mathrm{M}$. Barbuto et al. reviews the possibility to design metamaterial-inspired particles suitable for the signal manipulation in horn antennas. These particles, realized through either bi-omega or complementary split ring resonators, provide new degrees of freedom in the manipulation of the transmitted/received signal by horn antennas. In addition, they are able to eliminate the out-of-band noise and to change at will the polarization of the field radiated by these antennas. These two remarkable effects are obtained without the necessity of external devices but exploiting electrically thin structures to be inserted inside the antennas and realizable with traditional PCB techniques.

*e-mail: christos.argyropoulos@unl.edu; alessio.monti@unicusano.it

This is an Open Access article distributed under the terms of the Creative Commons Attribution License (http://creativecommons.org/licenses/by/4.0), which permits unrestricted use, distribution, and reproduction in any medium, provided the original work is properly cited. 
The paper by K. Achouri et al. is focused on the design and experimental realization of electromagnetic metasurfaces. In the beginning, the paper presents a brief overview of a design technique for electromagnetic metasurfaces based on the generalized sheet transition conditions and shows how this technique allows to determinate the exact tensorial surface susceptibility function. Then, the paper focuses on the design of a metasurface composed by both electric and magnetic scatterers. The paper concludes with a detailed analysis of the possible applications of metasurfaces according to their design complexity order. Among the different remarkable structures, an electromagnetic non-nonreciprocal non-gyrotropic metasurface has been introduced and discussed.

The paper by O. Vacus and C. Saint-Flour presents an investigation about the use of effective medium approximations for the computation of the radar cross section of spheres and cone-spheres with plasmonic coatings. It is shown that the results obtained using this approach exhibit a good agreement with the exact computation in a broad frequency range allowing a considerable saving of computational resources and time in many applications. Finally, the paper reports a notable application that is the possibility to use properly designed plasmonic-like coatings as an alternative to radar absorbing materials in anechoic chamber for radar cross section measurements.

The paper by A. Boardman et al. focuses on hyperbolic metamaterials and presents new outcomes for the definition of a new generalized theory about these structures topology. Here, the emphasis is put on the orientation of the optic axis that is a significant element in the optical properties of the material. It is also shown that, differently from conventional anisotropic waveguides, for a hyperbolic one a critical angle exists for which the guided waves are homogeneous for all the values of the polar angle. This remarkable effect facilitates the achievement of propagating modes in meta-waveguides and make the resulting structures more tolerant to optic axis misalignment.

In the paper by B. Orazbayev et al., a discussion about the design and performance of metalenses is proposed. The paper proposes the design of a low-profile Wood zone plate metalens based on fishnet metamaterials and working in a near-zero regime. This structure is able to ensure the low profile of the lens while exhibiting low reflection losses. An example of these interesting structures is designed and realized to operate in the $\mathrm{W}$ band. The measured properties show the possibility of improving focusing and radiation performance using the proposed design.

In the paper by R.Ø. Thorsen and S. Arslanagic, the focus is on the effect of the eccentricity on the core-shell active cylindrical nanoparticles. The paper shows that the displacements of the silver core relative to the silica shell strongly affect the super-resonances of the nanoparticles. This investigation, conducted for both small and large cores, plays an important role in the correct interpretation of the experimental results that are inevitable affected by small inaccuracies occurring during the fabrication process.

The paper by S.K. Patel and C. Argyropoulos contains a review of several linear and non-linear applications of nanoantennas. Among the different interesting applications discussed in this paper, it is shown that the scattering of a linear nanoantenna can be tuned to design robust optical wireless links. Moreover, the nonlinear and photodynamic responses of different nanoantennas are discussed and the authors envision the design of unconventional optical devices such as low-power nanoswitches, nanosensors and efficient energy harvesting devices.

The paper by T.A. Morgado and M.G. Silveirinha presents a theoretical study of the emission from a short horizontal dipole in the wire medium. The authors use an effective medium approximation allowing for explicit analytical results for the emission pattern. The derived theory demonstrates that, as a consequence of the ultrahigh density of photonic states of the nanowire array, the power radiated by the dipole is strongly enhanced compared to that emitted in the dielectric host material.

In the paper by M. Schaeffer and M. Ruzzene, an investigation of the equivalent in-plane mechanical properties of one dimensional and two dimensional periodic magneto-elastic lattices is reported. A lumped parameter model is used to describe the lattices using magnetic dipole moments in combination with axial and torsional springs. It is shown that lattices can be stiffened or softened and the Poisson's ratio can be tuned with these configurations.

The paper by Z. Li and K. Aydin presents designs of broadband metasurfaces for anomalous transmission and spectrum splitting at visible frequencies. In particular, the authors have numerically proposed a transmission-mode metasurface design realized with the quasi-continuous trapezoid-shaped nanoantenna arrays. By gradually modulating the phase shift along the interface, the metasurface could enable broadband anomalous transmission and spectrum splitting functionalities. The reported results will be of interest for applications in integrated nanophotonics.

B.M. Adomanis et al. report in their work three dimensional metamaterial unit cells using membrane projection lithography, a novel experimental technique used to fabricate a silicon matrix decorated with metallic inclusions. The proposed structures are characterized by FTIR spectroscopy and the results are in good agreement with corresponding simulations. Employing additional associated simulations the authors show that a slight change in the fabrication conditions can lead to significant changes in the electromagnetic response of the structures.

The paper by Y. Ra'di et al. studies wave propagation through non-reciprocal bi-anisotropic media, and in particular Tellegen and moving media in the limiting case of nihility, which are media with zero permittivity and permeability tensors. It is demonstrated that the moving nihility materials provide the extreme asymmetry in the phase shift of transmitted waves propagating along the opposite directions. In addition, the possibility to create an angular filter with extreme sensitivity to the incidence angle is revealed.

T. Brunet et al. paper provides an insightful diagrammatic representation of the sign of the index of refraction based on the real and imaginary part of its constitutive parameters. This study is done in the cases of both electromagnetic and acoustic metamaterials. It is shown that the double negativity required for perfect (non-dissipative) 
media is not necessary for real passive (lossy) metamaterials, for which single negativity of one of the two constitutive parameters may be sufficient.

In the paper by B. Ungureanu et al., the authors present new auxetic elastodynamic metamaterial designs that could lead to earthquake protection. It is numerically demonstrated that the propagation of seismic waves with different frequencies can be influenced by a meter scale version of these auxetic metamaterials buried in the soil. This novel class of seismic metamaterials opens band gaps at frequencies compatible with seismic waves and this response makes them interesting candidates for seismic isolation structures.

The paper by Yang et al. propose a novel perspective on complete absorption of sound waves at low frequencies. The authors demonstrate that the effect of coherent perfect acoustic absorption is a consequence of the geometric framework, similar to the electromagnetic case. Their analysis is based on Greens functions and comparisons between simulations and experiments for a specific unit cell design are provided.

Finally, the paper by G. Kenanakis et al. presents a review of the polarization control capabilities offered by different $\mathrm{THz}$ and far-IR chiral and bianisotopic metamaterial designs. The reported structures show large optical activity, tunable/switchable wave ellipticity, and polarization-dependent asymmetric transmission. The authors envision the realization of both passive and active $\mathrm{THz}$ polarization manipulation components like polarization filters, modulators, and waveplates.
Acknowledgements. We are particularly grateful to the Editor-inChief of the EPJ AM Journal Prof. Yang Hao (Queen Mary, University of London, London, United Kingdom) and to the General Chairs of the Metamaterials congress Prof. Filiberto Bilotti (Roma Tre University, Rome, Italy) and Prof. Andrea Alù (University of Texas at Austin, Austin, TX, USA) for establishing the Special Issue of the Metamaterials congress and giving us the opportunity to serve as Guest Editors in this first edition. We strongly believe that this Special Issue represents a diverse part of the work presented in the Metamaterials congress.

We would also like to sincerely thank all the authors that have enthusiastically accepted our invitation to submit a contribution to the first edition of this Special Issue. We believe that all the published papers meet the criteria of high-quality and high-impact that we have looked for during the selection process.

In addition, we thank all the reviewers that have been involved in the review process of this Special Issue. Their promptness and their meticulous work have allowed to further increase the quality of the published works and made possible to publish this Special Issue before the end of the year becoming available a short time after the congress.

We also would like to thank Ms. Ariana Fuga, publishing editor of the journal, and Ms. Catherine Brassac, Manuscript Management System Product Manager. Their punctual help has been crucial for the successfully publication of this Special Issue.

Finally, we would like to thank all the people interested in this Special Issue. We sincerely hope you will find interesting the variety of the representative published papers. We are looking forward to see you in the next edition of the Metamaterials Congress that will take place in Crete (Greece) on 17-22 September 2016.

Cite this article as: Argyropoulos C \& Monti A: Editorial to the topical issue "Advanced Metamaterials in Microwaves, Optics and Mechanics". EPJ Appl. Metamat. 2015, 2, 1. 


\section{Guest Editors}

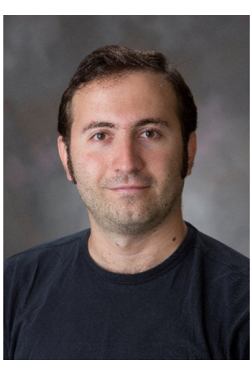

Christos Argyropoulos

University of Nebraska-Lincoln,

Department of Electrical and Computer

Engineering,

SEC, Room 239N, 844 N. 16th St., Lincoln, NE 68588-0511, USA

e-mail: christos.argyropoulos@unl.edu

URL: http://argyropoulos.unl.edu/

Christos Argyropoulos received the Diploma of Electrical and Computer Engineering from the Aristotle University of Thessaloniki, Greece (2006). He holds a M.Sc. degree in Communication Engineering from the Microwaves and Communication Systems group of the University of Manchester, UK (2007) and a Ph.D. degree in Electronic Engineering from the Antennas and Electromagnetics Group of the Queen Mary, University of London, UK (2011). After completion of his Ph.D. studies, he accepted a Postdoctoral Fellowship position in the University of Texas at Austin, USA. Next (2013), he worked as a Postdoctoral Associate in the Center for Metamaterials and Integrated Plasmonics at Pratt School of Engineering, Duke University, USA. From September 2014, he is an Assistant Professor at University of Nebraska-Lincoln, Department of Electrical Engineering, where he established the Metamaterials and Integrated Nanophotonics Lab.

He has published over 120 technical papers in highly ranked journals and refereed conference proceedings, including five book chapters. His main research interests include linear and nonlinear plasmonics, active metamaterials, computational electromagnetics, numerical and analytical modeling of metamaterials and their applications, novel antenna design, transformation optics, thermal emission from plasmonic structures, graphene nanophotonics, new energy harvesting devices and acoustic metamaterials. He has received several travel and research awards, such as Junior Researcher Award of the 2013 Raj Mittra Travel Grant, EPSRC Research Scholarship, Royal Academy of Engineering international travel grant and twice the Marie Curie Actions Grant to attend the European School of Antennas. He chaired a session at APS March Meeting 2014 and is the organizer of a special session at META 15 and META 16, respectively. He has given several invited talks and seminars to different conferences and universities. He serves as Student Paper
Competition co-chair at IEEE APS 2016 and as guest coeditor at the EPJ Applied Metamaterials special issue of the Metamaterials' Congress. He is a member of IEEE, IEEE Antennas and Propagation Society, Optical Society of America, SPIE, American Physical Society and the Technical Chamber of Greece.

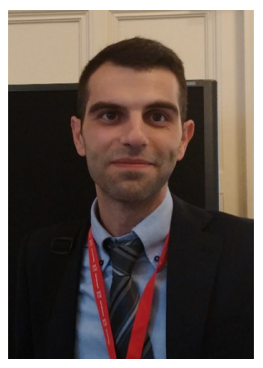

Alessio Monti

Assistant Professor

Niccolò Cusano University,

Via don Carlo Gnocchi 3, 00166 Rome, Italy

e-mail: alessio.monti@unicusano.it

URL: http://sites.google.com/site/

montialessio/

Alessio Monti was born in Rome, Italy, on February 16, 1987. He received the B.S. degree (summa cum laude) and the M.S. degree (summa cum laude) in electronic and ICT engineering both from Roma Tre University, Rome, in 2008 and 2010, respectively. From 2011 to 2013, he attended the doctoral school in biomedical electronics, electromagnetics and telecommunications engineering at Roma Tre University. Currently, he is an assistant professor at Niccolò Cusano University, Rome, Italy.

Dr. Monti served and serves as a member of the Technical Program Committee of several international conferences, as the 2016 IEEE International Symposium on Antennas and Propagation and the International Congress on Advanced Electromagnetic Materials in Microwaves and Optics (since 2014). He has also been serving as a Technical Reviewer of the many high-level international journals related to electromagnetic field theory, metamaterials and plasmonics and has been selected as one of the Top Reviewers for the 2014/15 by the Editorial Board of the IEEE Transactions on Antennas \& Propagation.

His research interests include the design and the applications of microwave and optical artificially engineered materials and metasurfaces, the design of cloaking devices for scattering cancellation at microwave and optical frequencies with a particular emphasis on their applications to the antenna and sensor theory. Dr. Monti has been the recipient of some national and international awards and recognitions for his achievements, including the 2nd place (ex aequo) at the student paper completion of the conference Metamaterials'2012 and the Finmeccanica Group Innovation Award for Young people in 2015. 